Meta

Journal des traducteurs

Translators' Journal

\title{
La communication dans les organisations internationales : perception et reconstruction du discours
}

\section{Egan Valentine}

Volume 46, numéro 4, décembre 2001

URI : https://id.erudit.org/iderudit/004520ar

DOI : https://doi.org/10.7202/004520ar

Aller au sommaire du numéro

\section{Éditeur(s)}

Les Presses de l'Université de Montréal

\section{ISSN}

0026-0452 (imprimé)

1492-1421 (numérique)

Découvrir la revue

\section{Citer cet article}

Valentine, E. (2001). La communication dans les organisations internationales : perception et reconstruction du discours. Meta, 46(4), 699-710.

https://doi.org/10.7202/004520ar
Résumé de l'article

La reconstruction du discours (traduction-rédaction) exige principalement des connaissances linguistiques et extralinguistiques ainsi que la capacité d'analyse et de synthèse. Dans le cadre de débats au sein d'organismes internationaux, où l'on discute de sujets politiques délicats ou de proposition d'actes juridiques complexes, s'ajoutent à ces exigences, une bonne capacité d'écoute, la compréhension des enjeux ainsi qu'un certain sens de la diplomatie. En nous fondant sur notre expérience, nous nous proposons d'examiner les conditions de construction du discours dans cette situation de communication particulière et de dégager certains principes qui sous-tendent sa production. 


\title{
La communication dans les organisations internationales: perception et reconstruction du discours
}

\author{
EGAN VALENTINE \\ Université du Québec à Trois-Rivières, Trois-Rivières, Canada
}

\begin{abstract}
RÉSUMÉ
La reconstruction du discours (traduction-rédaction) exige principalement des connaissances linguistiques et extralinguistiques ainsi que la capacité d'analyse et de synthèse. Dans le cadre de débats au sein d'organismes internationaux, où l'on discute de sujets politiques délicats ou de proposition d'actes juridiques complexes, s'ajoutent à ces exigences, une bonne capacité d'écoute, la compréhension des enjeux ainsi qu'un certain sens de la diplomatie. En nous fondant sur notre expérience, nous nous proposons d'examiner les conditions de construction du discours dans cette situation de communication particulière et de dégager certains principes qui sous-tendent sa production.
\end{abstract}

\begin{abstract}
Language professionals involved in summary and report writing in international organisations require not only translation skills, the capacity for analysis and synthesis and knowledge of the issues, but also "diplomatic" skills. This article looks at the task of the summary writer, the demands of this particular communication situation, and the principles underlying the summary writing process.

MOTS-CLÉS/KEYWORDS

communiqués, organisations internationales, reconstruction du discours, résumé
\end{abstract}

\section{Introduction}

Dans la communauté des nations, la communication est à la fois un instrument stratégique et un espace multidimensionnel. Elle est l'outil et le théâtre d'une nécessaire interaction de plusieurs langues, d'intérêts divergents et d'idéologies opposées qui conduisent à une actualisation particulière du discours et sollicite, chez le langagier œuvrant dans ce contexte, de multiples compétences bien spécifiques.

En effet, au sein des assemblées internationales, où les représentants s'expriment au nom de leurs États respectifs, le terme communication renvoie non seulement aux échanges linguistiques entre interlocuteurs (ici les orateurs) au cours des délibérations, mais aussi aux messages écrits (rapports, comptes rendus, communiqués de presse) auxquels donnent lieux ces échanges ainsi qu'aux critères qui guident la rédaction de ces écrits. Le langagier est alors appelé à remplir diverses fonctions «décloisonnées » : traduction, interprétation, prise de notes, rédaction de résumés et de rapports, révision. Pour ce faire, il doit mobiliser simultanément des compétences linguistiques, la capacité d'analyse et de synthèse et des aptitudes techniques (et la capacité d'acquérir de nouvelles connaissances et de les mettre en œuvre rapidement). 
Dans le cas du rédacteur de résumés et de comptes rendus au sein d'organismes internationaux (résumeur-rédacteur), s'ajoutent à cette multicompétence le jugement (c'est-à-dire la capacité de filtrer l'information et de faire rapidement la part entre l'essentiel, l'utile et l'accessoire), ainsi qu'un certain sens ou plutôt un sens certain de la diplomatie. Les fonctions évoquées plus haut visent à s'assurer que les écrits produits reflètent, dans la mesure du possible, la réalité des délibérations et la nature d'un discours juridico-politico-technico-diplomatique, à la fois privé et public, polémique et conciliant.

Dans cet article, nous proposons un ensemble de réflexions et d'observations nées, d'une part, de notre pratique de la traduction et de la rédaction de résumés et, d'autre part, de notre intervention auprès de deux organismes internationaux à titre de responsable de la rédaction de résumés et de comptes rendus des délibérations.

Résolument descriptive, notre démarche vise à optimiser, à travers l'objectivation d'une activité de rédaction, le rôle du langagier appelé à suivre les débats, à les transcrire et à les résumer pour diffusion presque immédiate. Cette activité s'inscrit donc dans la trilogie traduction-résumé-rédaction, mais d'une manière inhabituelle: elle fait simultanément appel à des compétences traductionnelles et à la capacité de synthétiser des discours et de manier les ressources de la langue à différents niveaux.

Nous mettrons en évidence, les divers éléments qui contribuent à ce savoir-faire dans ce contexte en nous appuyant sur un corpus de textes émanant de deux organismes internationaux soit l'Autorité internationale des fonds marins (ISA) et le Programme pour l'environnement des Caraïbes (PEC).

\section{Les organismes}

Créée en 1994, en application de la Convention des Nations unies sur le droit de la mer de 1982 et à l'Accord de 1994 relatif à l'application de la partie XI de ladite Convention, l'Autorité internationale des fonds marins (ISA), qui compte 135 État membres, a pour mission d'organiser et de contrôler, pour le compte des États parties à la Convention, les activités menées aux fins d'exploration et d'exploitation des ressources des fonds marins et de leur sous-sol, au-delà de la limite de la juridiction nationale. La Convention établit un régime international pour la gestion des ressources minérales du fond des océans, ressources considérées comme le patrimoine commun de l'humanité.

Depuis quelques années, les travaux du Conseil de l'Autorité ont porté principalement sur l'examen, article par article, du code d'exploitation minière qui régit les activités menées dans la zone. Ce code énonce en détail, tant du point de vue juridique que pratique, les procédures devant régir la prospection et l'exploration des nodules polymétalliques dans la zone internationale des fonds marins. Il régit la façon dont les parties intéressées peuvent devenir contractantes en obtenant de l'Autorité l'approbation de leurs plans de travail touchant l'exploration de telle ou telle zone géographique.

Le Programme pour l'environnement des Caraïbes du PNUE (PEC), s'occupe, lui, de questions environnementales. Il s'agit d'un programme intergouvernemental pour le développement durable des ressources marines et côtières de la région des Caraïbes. La Convention pour la protection et la mise en valeur du milieu marin 
dans la région des Caraibes et ses protocoles constituent les instruments qui fournissent le cadre juridique pour l'élaboration et la mise en œuvre du Programme. Des réalisations récentes du PEC ont été l'adoption du Protocole relatif à la pollution due à des sources terrestres dans le cadre de la Conférence des plénipotentiaires et la tenue de la neuvième réunion intergouvernementale sur le plan d'action du Programme pour l'environnement des Caraïbes et de la Sixième réunion des parties contractantes à la Convention pour la protection et la mise en valeur du milieu marin dans la région des Caraïbes.

\section{Complexité du rôle du langagier et polyvalence nécessaire}

La présentation sommaire de ces deux organismes montre la spécificité du contexte dans lequel œuvre le langagier. Mais d'autres contraintes s'y rattachent. Le langagier se trouve, en effet, au milieu de conflits qui le dépassent: querelles idéologiques, conflits d'intérêts évidents résultant des rapports de force et de pouvoir: opposition Nord-Sud, entre pays en développement et pays développés, entre Occident et Orient, sans oublier les jeux de coulisse. Le langagier se trouve dans un univers qui a ses codes et conventions qu'il doit comprendre et traduire, un univers empreint de cérémonial et de formalisme protocolaire qu'il doit nécessairement appréhender et apprivoiser.

Trois grands critères guident, à notre sens, le travail du rédacteur-résumeur au sein des assemblées internationales. Nous avons dégagé ce que nous avons choisi d'appeler la règle des trois $\mathrm{D}$ de la rédaction des écrits: a) discernement, c'est-à-dire l'esprit critique et la faculté d'apprécier avec justesse les situations et les choses; b) distanciation, c'est-à-dire le recul par rapport aux événements, et c) diplomatie, c'est-à-dire l'art d'exprimer avec discrétion, tact et habileté. Ces catégories ne sont évidemment pas étanches, et, comme nous le verrons, des recoupements existent.

\section{Discernement}

Le discernement fait appel avant tout au jugement, mais discernement et distanciation s'opposent parfois et se rejoignent souvent. Autant il peut être nécessaire de ne pas fournir toutes les traces d'une intervention, si la situation l'exige (situations de conflit évident), comme par exemple lors de délibérations officieuses, autant il peut être nécessaire de souligner la contribution de tout un chacun dans d'autres situations.

L'exemple qui suit illustre la décision avisée du rédacteur de citer nommément les intervenants lors d'un débat portant sur l'état des travaux relatifs à l'élaboration du code d'exploitation minière. La situation et la tonalité générale des interventions, parfois musclées, mais toujours nuancées, autorisent à agir ainsi. Cette décision permet aussi de mettre en valeur l'apport des intervenants de toutes les régions géographiques sans évoquer la moindre controverse:

\footnotetext{
À l'issue de la séance de ce matin, le Secrétaire général a fait observer que le Conseil avait réalisé des "progrès substantiels» dans l'ensemble, et que les résultats obtenus jusqu'ici inspiraient un certain optimisme. Quelques délégations, cependant, ont exprimé de l'insatisfaction et une certaine inquiétude face à la lenteur des travaux.
} 
Concernant les progrès réalisés, l'Allemagne a déclaré que l'élection du Secrétaire général était, à son avis, le seul résultat tangible de la session: malgré le compromis que cette délégation avait tenté de proposer, le manque de progrès relatif au code minier n'était pas de bon augure pour la deuxième partie de la session en juillet. [...]

La Jamä̈que, prenant la parole au nom du Groupe d'Amérique latine et des Caraïbes, a souligné l'importance pour son groupe que l'on garantisse la protection et la préservation du milieu marin [...]. Cette délégation a déploré le fait que des questions délicates évoquées par le secrétariat n’aient pas été réglées malgré deux semaines de débats. [...]

La République de Corée a déclaré que des progrès avaient été réalisés sur les principales questions du code minier, vu que les délégations avaient pu identifier plus clairement les points d'achoppement sur certaines questions-clés.

Le Nigéria, au nom du Groupe africain, a signalé les difficultés que représentait la tâche de trouver un équilibre entre les différents intérêts en jeu, à savoir, la protection des investissements et la protection du patrimoine commun de l'humanité. Malheureusement, «nous n'avons pas fait beaucoup de progrès».

Au nom du Groupe d'États d'Europe orientale, la République tchèque a déclaré que son groupe n'avait pas perdu l'espoir pour que l'examen du code d'exploitation minière soit achevé en juillet. [...]

(Communiqué de presse FM/6/16 - 31 mars 1999.)

Par contre, dans les cas suivants où il s'agit de situations conflictuelles, les rédacteurs ont décidé, à juste titre, de ne pas identifier les délégations ou les personnes concernées par le débat. Dans le premier cas, la discussion porte sur l'article 32 du code qui concerne les actions à prendre en cas de dégâts au milieu marin :

L'article 32 a suscité un grand nombre d'interventions. Cet article avait déjà fait l'objet d'un examen attentif par le Conseil à la session de 1999. Nombre de délégations ont fait part de leur insatisfaction à l'égard de la rédaction du texte. À leur avis le texte ne donne pas un mandat clair et définitif au Secrétaire général; certaines parties demeurent obscures. Il a été demandé une reformulation de ce texte afin de tenir compte des préoccupations de toutes les délégations.

(Communiqué de presse FM/6/27 - 13 juillet 2000.)

Dans le deuxième cas, il s'agit d'un désaccord au sein de la Commission juridique et technique, un des organes subsidiaires de l'Autorité des fonds marins:

L'un des membres de la Commission n'a pas participé au processus de sélection, tandis qu'un autre membre s'est élevé contre le fait qu'il n'était pas conforme à celui du programme de formation de la Commission préparatoire. Le Président de la Commission a demandé aux membres intéressés de la Commission juridique et technique de présenter des propositions quant à la nature et aux objectifs des futurs programmes de formation de l'Autorité.

(Rapport de la Commission juridique et technique 1998.)

L'exemple qui suit est un texte laudatif qui résume les hommages rendus, en début de séance, par les membres de l'Assemblée lors du décès d'un ancien membre de la délégation des États-Unis. Vu la nature de l'événement, le statut du diplomate en question, le nombre et la teneur des témoignages, les rédacteurs ont cherché à rendre toute l'importance et l'émotion perçues au sein de l'assemblée. À noter également, le 
souci de la part de la rédaction de refléter, dans le discours rapporté, une représentation géographique équitable des membres de l'Assemblée.

\section{Hommage à M. Elliott Richardson}

Dans l'hommage rendu à M. Richardson, l'observateur des États-Unis a retracé la brillante carrière de ce dernier qui avait été, à quatre reprises, ministre sous divers présidents et qui avait mérité la plus haute distinction de son gouvernement. Soulignant le profond intérêt de $\mathrm{M}$. Richardson pour le droit de la mer, la représentante a signalé que ce dernier avait toujours cru aux bases sur lesquelles reposent le droit de la mer et qu'il n'avait jamais perdu de vue l'objectif visé par la Conférence sur le droit de la mer.

La Fédération de Russie a souligné la contribution importante de M. Richardson à la partie XI de la Convention, partie grâce à laquelle l'Autorité a été créée.

La République de Corée, au nom des pays du Groupe des États d'Asie, a exprimé sa profonde tristesse à la disparition de cet homme qui avait consacré sa vie au service des intérêts de son pays et à l'édifice qu'est l'Autorité internationale des fonds marins.

Au nom du Groupe des États d'Afrique, le Nigéria a qualifié M. Richardson de diplomate hors pair et de combattant, un homme de grand courage et d'une parfaite intégrité.

Prenant la parole au nom du Groupe des États d'Amérique latine et des Caraïbes, le Mexique a déclaré qu'il fallait classer M. Richardson parmi les grands diplomates et associer son nom aux pères fondateurs de l'Autorité.

La délégation d'Australie a souligné les talents de diplomate chez M. Richardson qui a su mettre les intérêts de son pays avant les siens.

Le Secrétaire général a salué en M. Richardson non seulement les qualités professionnelles, mais aussi les qualités humaines: promoteur de la coopération internationale et du multilatéralisme, M. Richardson avait déployé des efforts inlassables pour le droit de la mer et avait tout fait pour inciter son pays à adhérer à la Convention. Négociateur habile, il a su allier professionnalisme, bonhomie, charme et cordialité.

(Communiqué de presse $\mathrm{FM} / 6 / 10-23$ mars 2000)

Comme on peut le voir, le discernement comprend jugement et prudence, astuce et circonspection, sagacité et flair.

\section{Distanciation}

Qu'il s'agisse de faits, de réalité ou d'opinions, une certaine distance s'impose afin d'assurer la neutralité et l'objectivité voulues dans le travail de rédaction. Cette distanciation, pas toujours facile à réaliser, ne doit pas pour autant nuire à l'intention générale du message ni à son contenu informatif. Elle est souvent manifestée par le recours à l'anonymat quant à l'identité ou la provenance géographique de l'orateur, la dépersonnalisation du discours par le recours à la voix passive, par exemple. C'est ce qui se produit d'ailleurs dans le cas des communiqués de presses rédigés à l'issue des discussions officieuses. Les extraits suivants montrent comment la rédaction peut veiller à la neutralité — anonymat surtout, avant des prises de position définitives des participants.

Le premier exemple rapporte une partie de la discussion relative à la question de confidentialité, question litigieuse qui avait été longuement débattue au sein du Conseil de l'Autorité. Un des articles du code minier prévoit que les données et les 
informations « ayant une valeur commerciale » doivent rester confidentielles pendant une certaine période de temps. Les avis sont très partagés, tantôt quant à la terminologie ou à la formulation linguistique, tantôt quant à la durée de la confidentialité:

Nombre de délégations se sont interrogées sur l'emploi du terme "valeur commerciale», considéré comme vague et mal défini et en ont demandé la suppression.

D’autres trouvaient ce terme étrange. Certaines délégations lui préféraient le terme «droit de propriété industrielle». L'emploi du terme « information sensible» a été remis en question.

À propos de la question de la durée de la période de confidentialité, nombre de délégations ont demandé que la période de dix ans soit réduite, de manière à permettre, dans l'esprit de la démocratie, à d'autres entités de profiter des informations concernant les richesses disponibles dans les fonds marins.

Une délégation a insisté pour que la période soit fixée à 3 ou 5 ans; encore une autre a proposé que l'on lie cette durée à celle du plan de travail qui, lui, est fixé à 5 ans. Encore une autre a demandé que la durée de la confidentialité ne soit pas limitée. Opposant cette idée, une autre délégation a fait observer qu'un tel changement serait contraire à la lettre et à l'esprit de la Convention qui traite de la facilitation du transfert de la technologie, surtout vers les pays en développement.

(Communiqué de presse FM/6/11 - 23 mars 2000.)

Cet exemple de distanciation est particulièrement intéressant. Il occulte des enjeux majeurs qui sous-tendent le débat: les pays développés possèdent la technologie et se montrent réticents à livrer certaines informations. Ils craignent, en outre, les incidences financières liées à la proposition des pays en développement voulant que le code prévoie, en cas de catastrophe, une compensation à l'endroit des États côtiers dont font partie certains pays en développement. De plus, certains pays développés sont aussi investisseurs pionniers. Ces enjeux majeurs, le résumeur doit les percevoir et les comprendre. En d'autres termes, il doit s'en rendre compte sans en rendre compte. Ajoutons que l'essentiel dans cette situation, c'est la résolution du problème et non le processus mouvementé qui conduit à sa résolution, comme le montre le passage suivant:

Des questions relatives à la confidentialité ont été traitées au cours des réunions du Conseil en mars dernier. Un consensus a été dégagé sur plusieurs articles visant à protéger les données et informations et à ne permettre leur utilisation par les personnel autorisé de l'Autorité «que dans la mesure nécessaire à l'exercice effectif de leurs attributions et fonctions».

La levée de la confidentialité ne se fera plus automatiquement tous les dix ans, mais fera l'objet d'une révision par le Secrétaire général et le contractant. Demeurent confidentielles les informations dont le contractant considère que leur divulgation serait «susceptible de lui causer injustement un grave préjudice économique». Aucune donnée ou information n'est divulguée avant que le contractant n'ait raisonnablement eu la possibilité d'épuiser les recours judiciaires. [...]

[...] le document nouveau représente une tentative d'établir un équilibre entre les considérations liées à la confidentialité et celles liées à la protection du milieu marin, le président a rappelé le devoir qui incombait aux délégations de poursuivre les travaux dans un esprit de coopération dans la recherche d'un compromis acceptable par tous.

(Communiqué de presse FM/6/29 - 14 juillet 2000.) 
Il arrive que le résumeur soit confronté à des situations où il doit faire abstraction de sa propre perception des choses ou de ses propres sentiments. Il doit taire ses partipris, ses propres valeurs; ses convictions ne doivent en aucun cas transparaître; autrement dit, il doit faire preuve de tact et d'habileté, éléments essentiels à la mâ̂trise du troisième $\mathrm{D}$, la diplomatie.

\section{Diplomatie}

Discernement et distanciation ne vont pas sans diplomatie, art qui requiert, entre autres, des dons d'observation, d'intuition et de discrétion. Ici, ce terme renvoie à l'habileté dans les relations textuelles. Le rédacteur doit être en mesure d'exprimer même ce qui paraît difficilement exprimable, sans froisser les sensibilités. Ce critère implique doigté et finesse, tact et adresse dans le discours. Les assemblées internationales sont souvent le lieu de controverses et de profondes divergences dont il faut rendre compte dans le respect du formalisme protocolaire qui est le propre de ces assemblées.

Le paragraphe suivant rapporte, avec beaucoup de retenue, une intervention colérique qui, dans les faits, a été beaucoup plus virulente que ne laisse paraitre le compte rendu. Il s'agit d'une altercation, au sujet de procédures, entre le chef d'une délégation et le Président du Comité plénier lors de la Conférence de plénipotentiaires pour l'adoption du Protocole relatif à la pollution due à des sources et activités terrestres dans la région des Caraïbes:

Un large consensus se faisant progressivement jour sur les principaux éléments du Protocole, le Président a déterminé la procédure à suivre pour faciliter la négociation des questions en suspens et mettre fin au débat. [...] La délégation colombienne a protesté énergiquement contre cette façon de procéder.

La délégation colombienne a reproché au Président de refuser systématiquement de la laisser formuler ses objections et, ce faisant, de la tenir à l'écart de façon fort peu diplomatique. Le Président l'a assurée qu'elle aurait la possibilité de se faire entendre et a insisté sur la nécessité de faire avancer le débat de façon constructive et sans délai pour permettre aux Parties d'atteindre leur objectif commun.

(Rapport du Comité plénier — par. 47-48.)

Nombre d'organisations internationales connaissent une situation financière critique. Dans bien des cas, leurs recettes ne suffisent pas au financement de leur budget ordinaire. Cette situation est en partie attribuable au fait que certains États membres ne versent pas leurs contributions dans les délais prescrits. Les assemblées, en comité plénier, ne cessent de rappeler aux membres leurs obligations financières envers l'organisation. Il s'agit d'une question préoccupante, mais délicate: comment remédier à la précarité persistante des finances sans réprimander nommément les mauvais payeurs?

L'exemple suivant montre comment cette situation a été présentée, diplomatiquement, dans le rapport de la Neuvième réunion intergouvernementale sur le Plan d'action du PEC et de la Sixième réunion des Parties contractantes à la Convention pour la protection et la mise en valeur du milieu marin dans la région des Caraïbes:

En ce qui concerne les finances, M. Andrade a souligné les difficultés auxquelles faisait face le PEC. Il a insisté auprès des pays sur la nécessité de verser leurs contributions. 
Le faible niveau des contributions reçues par le Secrétariat du PEC a été noté avec regret, et il a été rappelé que celles-ci restaient insuffisantes pour une dotation en personnel et une gestion adéquates de l'UCR.

M. Andrade a également déclaré que, bien que le Secrétariat ne tienne ni à rendre les contributions obligatoires ni à les augmenter, les gouvernements devaient impérativement verser leurs contributions annuelles dans les douze mois sans être sujets à une approche punitive.

(Rapport du Comité plénier — par. 30, 48, 54.)

Toujours, sur la question des finances, cet autre texte incitant les membres de l'Autorité internationale des fonds marins à payer leurs cotisations, rapporte subtilement ce qui s'est véritablement passé lors de l'Assemblée où le Secrétaire général avait été beaucoup plus incisif dans ses propos.

L'Assemblée a lancé un pressant appel aux membres et aux anciens membres provisoires pour qu'ils règlent, le plus rapidement possible, leurs contributions en souffrance. La Commission des finances a noté que les arriérés de contributions se chiffrent à 1,5 millions de dollars dont un montant de 1,3 millions remonte à 1998 .

(Communiqué de presse FM/6/19 - 3 juillet 2000.)

Dans les cas qui viennent d'être cités, discernement et distanciation se conjuguent pour produire un discours diplomatique plutôt neutre. Par ailleurs, à la suite de tels rappels ou de telles exhortations, souvent les États visés ne tardent pas à exprimer devant l'Assemblée leur intention de s'acquitter le plus rapidement possible de leurs obligations.

Un autre exemple concerne une situation conflictuelle où la Fédération de Russie s'opposant à une proposition de l'Assemblée a clairement menacé de recourir à son droit de veto. Cet incident a été rapporté ainsi :

La Fédération de Russie, réagissant à la proposition des autres délégations, a évoqué la possibilité de se prévaloir de son droit de veto.

(Communiqué de presse FM/4/27 - 27 août 1998.)

Citons enfin les appels à la collaboration ou les cas d'interventions par de hauts dignitaires ou de membres haut placés de l'organisation. Le contexte exige que l'on en tienne compte tout en leur donnant l'importance jugée nécessaire. En plus d'exiger un esprit d'analyse et de synthèse aiguisé, ces résumés nécessitent parfois une grande dose de grandiloquence. Voici l'exemple d'une exhortation à la collaboration et au compromis :

Au cours du débat approfondi qui a suivi, une nécessité a été constamment mise en avant, celle de trouver un terrain d'entente, de telle façon que le Protocole soit réaliste et viable. Le représentant du Secrétariat a rappelé aux délégués que la détérioration de l'environnement porterait préjudice aux économies de la région. Bien que toutes les délégations aient le même objectif, protéger et préserver le milieu marin, elles étaient également conscientes du défi gigantesque qui leur était posé : obéir à la volonté politique et à celle du public et trouver un équilibre entre les idéaux du Protocole et les réalités économiques et politiques de la région de Caraïbes.

(Rapport du Comité plénier du PEC, LBSMP Protocol, par. 41.) 
L'exemple qui suit rend compte de l'intervention d'un ministre lors de la Neuvième réunion gouvernementale du PEC:

Les participants ont reconnu la présence du Ministre des Transports et des Communications des Antilles Néerlandaises, M. Adriaens, chef de la délégation du Royaume des Pays-Bas. Celui-ci a présenté une proposition officielle du Gouvernement des Antilles Néerlandaises pour l'accueil d'un Centre d'activités régional [...].Cette proposition a été suivie d'une présentation de REMPEITC et appuyée par l'OMI.

(Rapport de la réunion, par. 83.)

Les exemples qui suivent résument deux événements importants: la cérémonie de signature de l'Accord de siège et le résultat des négociations au sujet du code d'exploitation. Ces deux extraits illustrent le cérémonial propre à de telles manifestations et le discours de circonstance qui contribuent à magnifier les réalisations de l'organisation :

Signature de l'Accord de siège entre le gouvernement de la Jamaïque et l'Autorité internationale des fonds marins

Cet après-midi a eu lieu la signature de l'Accord entre le Gouvernement de la Jamaïque et l'Autorité internationale des fonds marins relatif au siège de l'Autorité.

M. Seymour Mullings, Premier Ministre adjoint et Ministre des Affaires étrangères et du Commerce extérieur, représentait le Gouvernement de la Jamaïque alors que l'Autorité était représentée par le Secrétaire général, M. Satya N. Nandan. La cérémonie s'est déroulée au Jamaica Conference Centre lors d'une séance de l'Assemblée de l'Autorité, convoquée pour cette occasion. L’Accord a été approuvé par l'Assemblée hier, 25 août.

Dans le discours qu'il a prononcé, $M$. Mullings a déclaré que la signature de l'Accord permettrait à l'Autorité de consacrer ses efforts et ses ressources à la réalisation de ses objectifs et fournirait, au Secrétaire général et à l'ensemble du personnel de l'Autorité, un cadre de travail agréable tout en garantissant la stabilité et la sécurité.

Réitérant l'attachement de son pays aux idéaux de la Convention de 1982 des Nations unies sur le droit de la mer, M. Mullings a qualifié la signature de l'Accord de nouveau jalon dans l'établissement de l'Autorité. Il a exprimé l'espoir que l'événement d'aujourd'hui donnera une impulsion favorable aux négociations nécessaires à l'élaboration du cadre institutionnel de l'Autorité.

Le Secrétaire général a évoqué les premières activités organisées à Kingston en relation avec le droit de la mer: la première session de la Commission préparatoire de l'Autorité internationale des fonds marins et du Tribunal international du droit de la mer, puis les réunions de l'Autorité depuis sa création en 1994, ont eu lieu ici même, sur le site devenu aujourd'hui le siège permanent de l'Autorité.

M. Nandan a exprimé l'espoir que les rénovations et améliorations prévues ne tarderaient pas à se réaliser.

Le Président de l'Assemblée José Luis Vallarta-Marrón (Mexique) s'est dit reconnaissant des négociations et de l'Accord auquel elles ont abouti. Il a réitéré l'importance que représente la signature de l'Accord pour l'Autorité.

(Communiqué de presse FM/5/29 - 26 août 1999.)

\section{Adoption par le Conseil du règlement pour l'exploration des nodules polymétalliques}

Le Conseil de l'Autorité internationale des fonds marins, réuni à Kingston cet aprèsmidi, a adopté par consensus des règles et règlements pour la prospection et l'explora- 
tion des nodules polymétalliques dans la zone internationale au-delà de la juridiction nationale des États. Des applaudissements retentissants, provenant des États membres et des observateurs réunis, se firent entendre au Jamaica Conference Centre à l'adoption du code minier. Cet événement a rappelé l'enthousiasme qui a marqué l'adoption de la Convention de 1982 des Nations unies sur le droit de la mer, il y a 18 ans, à Montego Bay, Jamaïque. Le nouveau règlement renforce le cadre juridique contenu dans ce traité afin de protéger les ressources des fonds de mer comme «l'héritage commun de l'humanité».

L'approbation du règlement a été possible grâce au compromis final qui a été dégagé, dans le cadre des négociations privées, sur la seule question restante, à savoir, quel type de garantie financière et technique serait fournie par les contractants afin de s'assurer que l'Autorité peut faire face à des dommages environnementaux causés par des accidents liés à l'exploration dans les fonds marins.

Avant l'action prise par le Conseil aujourd'hui, son président, M. Sakiusa A. Rabuka (Fidji), a annoncé aux délégués qu'ils participaient à un moment critique dans le destin de l'Autorité.

Le Secrétaire général, M. Satya N. Nandan a qualifié l'adoption du code minier par le Conseil d'événement historique dans la vie de l'organisation, qui servira de base à des rapports harmonieux entre les contractants et l'Autorité et donnera un effet pratique à la Convention. [...]

(Communiqué de presse FM/6/27 - 13 juillet 2000.)

Ces exemples montrent la nécessité de posséder une parfaite connaissance des organisations et de leur culture. Une telle connaissance est essentielle à la maîtrise de tous les paramètres de cette situation de communication particulière.

Il faut considérer aussi la dimension technique du discours dans ce contexte. En effet, si chaque organisation a sa propre culture, elle a aussi son propre discours spécialisé que le résumeur doit assimiler, s'approprier. Par exemple, il doit pouvoir composer avec les termes comme nodule polymétallique, déversements d'hydrocarbures, gisements cobaltifères, charge d'effluents, ainsi que le contexte d'utilisation de ces termes.

Signalons aussi, à titre d'exemple, un certain nombre de traits linguistiques récurrents inhérents à ce type de discours :

- Les formulations dites officielles (La Réunion a été ouverte...; la Conférence a convenu de... .; de l'avis des délégations...; le texte négocié a été renvoyé au comité de... , le Secrétariat a pris bonne note des commentaires formulés par...; la Réunion a été clôturée par... ).

- La dépersonnalisation du discours et la fréquence des tournures passives pour fin d'objectivité et de distance surtout dans le cadre de réunions officieuses (certaines délégations; il a été noté; il a été proposé...).

- La fréquence des tournures (bâties sur les termes tels que accord, entente, consensus, leurs synonymes et quasi-synonymes - mots-clés en situation de négociation) évoquant la recherche de relations harmonieuses et la volonté d'assurer la continuité des relations:

- Parvenir à un accord;

- Dégager un consensus;

- Un large consensus se faisant progressivement jour sur les principaux éléments;

- Se rallier à la majorité; 
- Resserrer la collaboration;

- Redoubler les efforts;

- Poursuivre un objectif commun;

- Trouver un terrain d'entente;

- Aplanir les difficultés;

- Régler les différends;

- Résoudre les questions restées en suspens.

\section{Conclusion}

En guise de conclusion, nous proposons le schéma ci-après qui résume les principales étapes du processus de communication.

Étapes de la production du discours

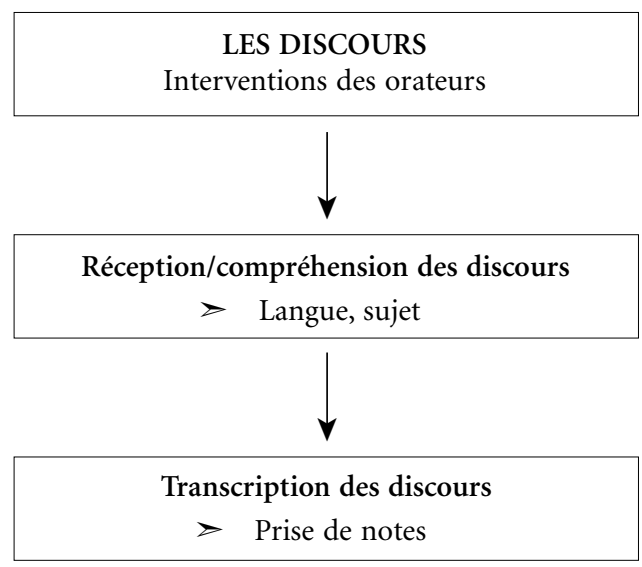

\section{Compétences}

$>$ Compétence linguistique

$>$ Connaissance du domaine

$>$ Jugement et tact

$>$ Connaissance des enjeux

\section{Critères}

$>$ Diplomatie

$>$ Discernement

$>$ Distanciation
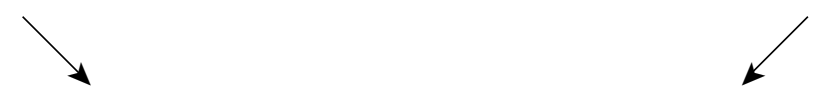

$$
\begin{array}{|c|}
\hline \multicolumn{1}{|c|}{\text { Filtrage - rédaction - discours }} \\
\qquad \begin{array}{l}
\text { Particularités du discours } \\
>\quad \text { Formes figées/formulations dites officielles } \\
>\quad \text { Dépersonnalisation du discours }
\end{array} \\
\hline
\end{array}
$$


Mettant en évidence l'interaction et l'interdépendance de tous les paramètres du contexte de communication, ce schéma montre les phases de la reconstruction des discours - depuis la réception jusqu'à la production du discours rapporté, en passant par l'opération de filtrage/reformulation.

En fondant notre réflexion sur une analyse interne et externe de la situation de communication, et à partir de l'examen des conditions qui ont donné naissance aux écrits, nous avons proposé des observations sur les écrits eux-mêmes.

Les étapes présentées dans cet article et résumées dans le schéma permettent d'assurer non seulement un discours bien adapté, mais aussi un discours acceptable, recevable, crédible et efficace. 